\title{
Implementation of Speed Variation in the Structural Dynamic Assessment of Turbomachinery Flow-Path Components
}

\author{
Andrew M. Brown, Ph. D \\ R. Benjamin Davis, Ph. D \\ Michael DeHaye \\ NASA/Marshall Space Flight Center \\ Propulsion Structural \& Dynamic Analysis
}

ASME Turbo Expo 2013

June 4, 2013, San Antonio, Texas 


\section{Introduction and Motivation}

- Structural ( $\mathrm{S}_{\mathrm{ult}}$ \& $\left.\mathrm{HCF}\right)$ assessment critical for turbomachinery flow path components undergoing possible resonance.

- Resonance generally avoided, but impossible for higher modes found with modern analysis, especially with wide speed ranges.

- Space Launch System upper stage J2-X Lox-H2 Engine Fuel Pump turbine stator operates from $26 \mathrm{Krpm}-34 \mathrm{Krpm}$; $69 \mathrm{~N}$ forcing excites modes $10-18$ between $30 \mathrm{KHz}-40 \mathrm{Khz}$.

- Criteria triggers forced response analysis at worst case resonant condition.

- Finite life analysis, where actual fatigue damage during operational time is calculated, frequently used if endurance limit criteria violated. 


\section{Many Turbopumps "Dither"}

- May be beneficial to incorporate fact that real turbopumps dither about a nominal mean speed.

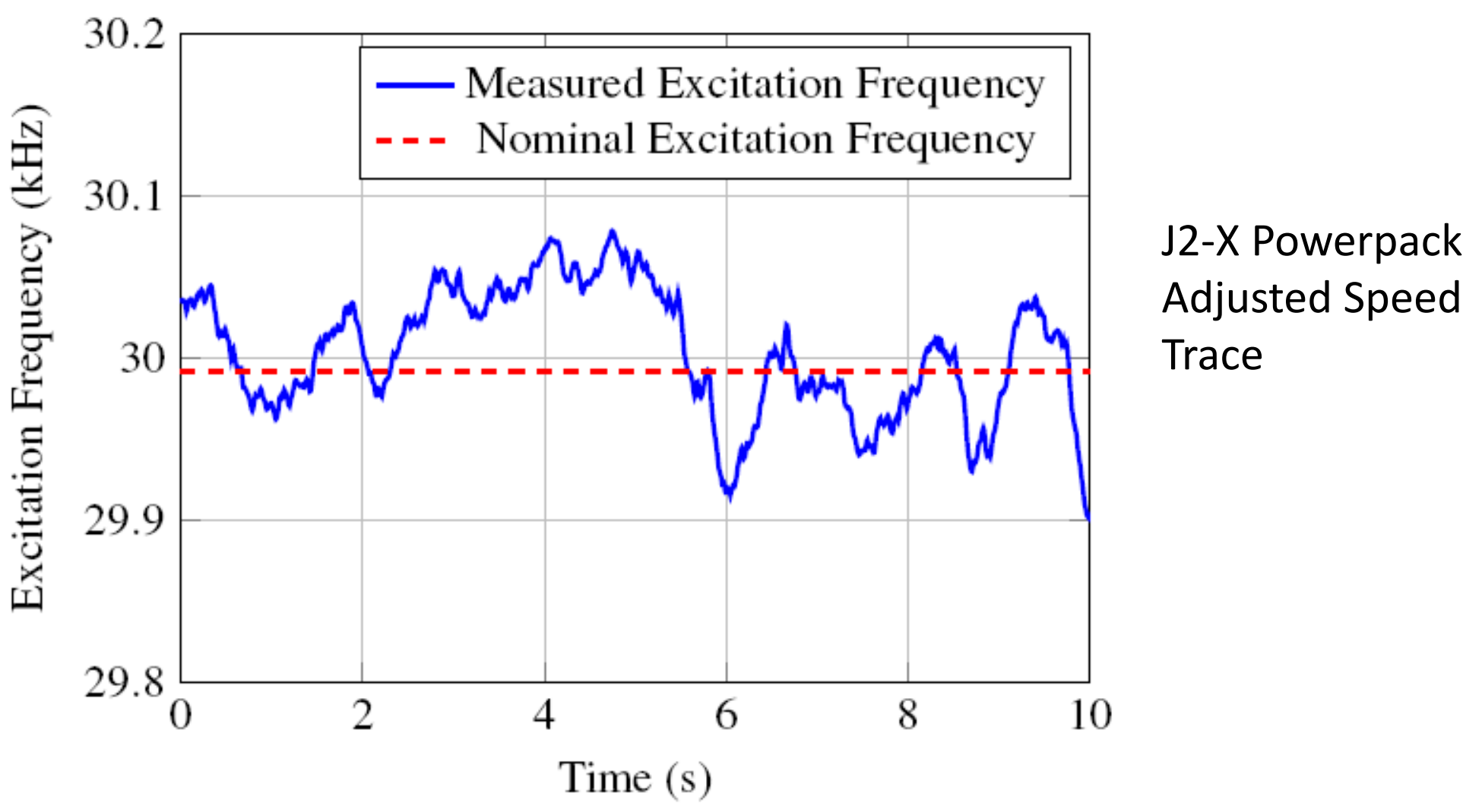

- During time speed is not exactly at natural frequency, damage accumulation is significantly reduced. 


\section{Agenda}

1. Introduction
a) Motivation
b) Literature Survey, Purpose Statement
c) Characteristics of Excitation Frequency

2. Theory and Development of Analysis Methods for Measured Speed Time Histories.
a) Numerical Method, Time Step Convergence Study
b) Analytical Method
c) Calculation of Dither Life Ratio

3. Monte Carlo Method for Unknown Speed History (Design).

a) Sensitivity to Damping, Speed standard deviation

4. Conclusion 


\section{Literature, Purpose}

- Intial studies of response of systems with time varying excitation frequency $\Omega$ by Lewis- 1932, Cronin- 1965.

- Lollack, 2002, defined reduction in peak response for monotically varying $\Omega$, useful for defining rate of sine-sweep tests.

- Henson, 2008, studied harmonically varying $\Omega$.

- For rocket engines, $\Omega$ varies non-deterministically. Motivated previous work by authors (2010) that developed numerical approach for calculating response and general sensitivities.

- Unacceptable HCF factor for $\mathrm{J2}-\mathrm{X}$ stator resonant $30 \mathrm{Khz}$ mode prompted need for practical technique.

- Purpose of this research

- to develop practical design techniques that account for excitation frequency stochasticity in the fatigue life of turbomachinery components. 


\section{Excitation Data}

- Taken from hot-fire testing of J2-X and SSME.

- $\Omega$ = engine speed ( $\mathrm{hz}$ )*[forcing pressure distortions/Rev] (FPR).

- Since purpose is to examine fatigue life at resonance, actual mean speed adjusted to natural frequency for analysis.

- Histograms for two different engines show Gaussian distribution of speed.
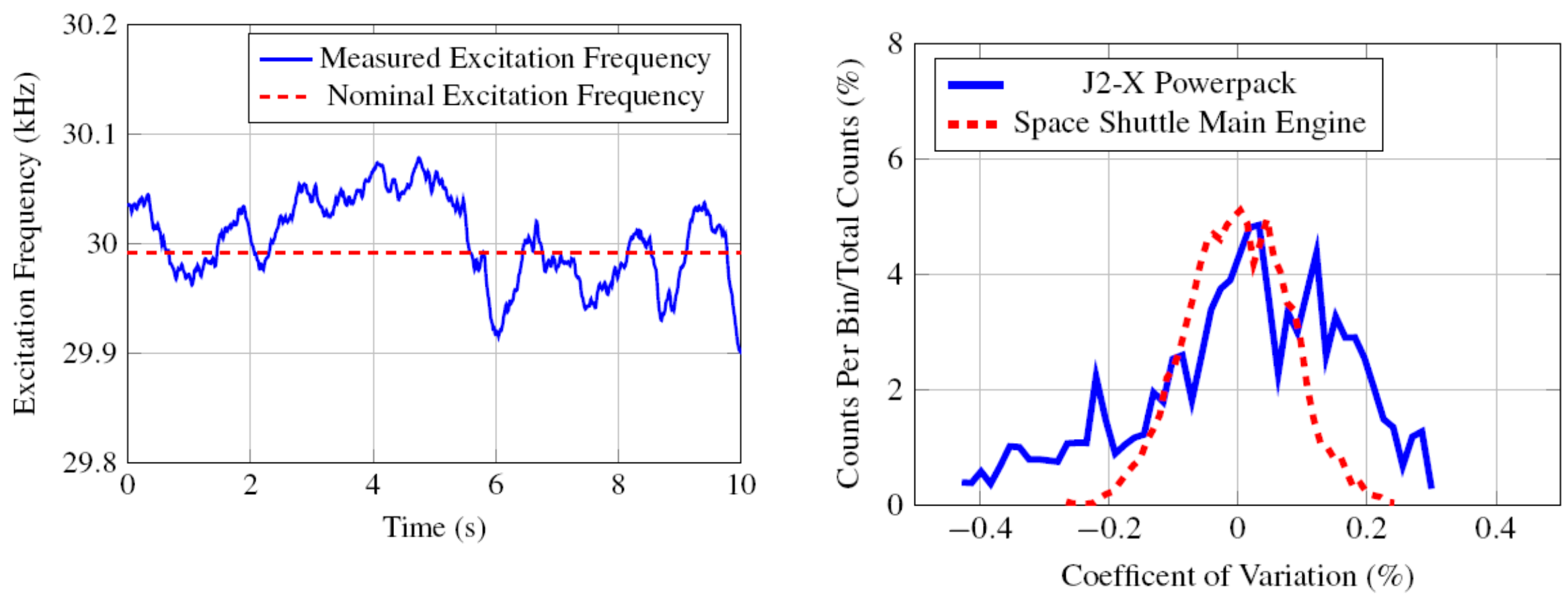
- SDOF EOM

$$
\begin{array}{r}
\ddot{x}+2 \varsigma \omega \dot{x}+\omega^{2} x=\frac{f(t)}{m} \\
\text { where } \quad f(t)=A \sin (\phi(t))
\end{array}
$$

- $\Omega$ is derivative of $\phi(\mathrm{t})$, constant in classical vibration analysis. For specified time-varying $\Omega$,

$$
\phi(t)=\int_{0}^{t} \Omega(\tau) d \tau
$$

- Calculate A necessary to generate peak resonant value of $\sigma_{\text {alt }}$ previously obtained by FEA,

$$
\sigma_{\text {alt }} \equiv x=\frac{A}{\omega^{2} 2 \zeta}
$$

- Now can solve for $\sigma_{\text {alt }}$ in EOM with using numerical Runge-Kutte procedure implemented in Matlab; agrees with Lollack's results for linearly varying $\Omega$. - Finally, Calculate damage fraction $\Phi$ using Miner's rule, $\Phi=\sum_{i=1}^{K} \frac{n}{N}$, which
becomes

$$
\Phi(t)=\int_{0}^{t} \frac{\Omega(\tau)}{N(\tau)} d \tau
$$


- Previous work indicated convergence at $\Delta t=1 / 40 f_{n}$.

- Initial studies here showed high freqency oscillation, so response and damage convergence studies performed $\rightarrow \Delta t=1 / 120 f_{n}$.
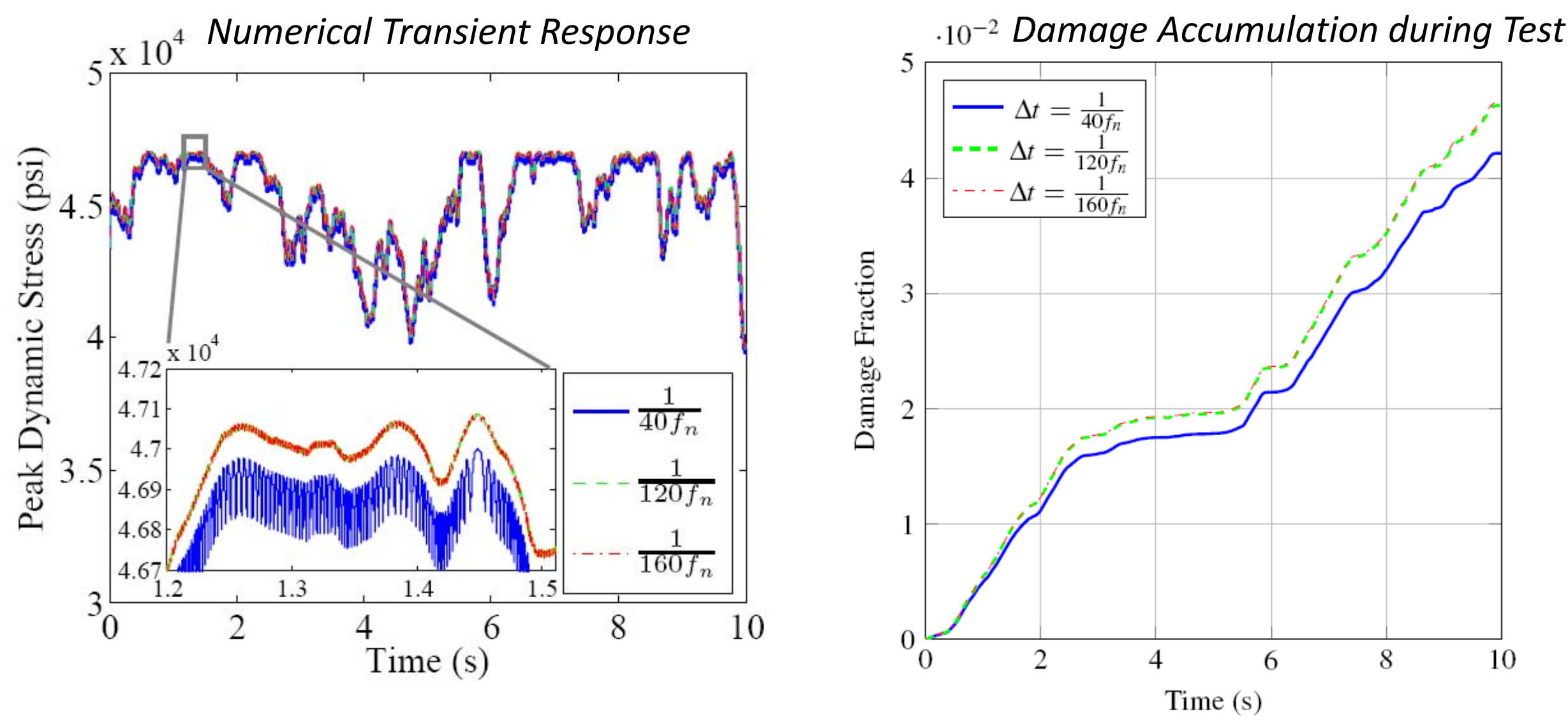


\section{Analytical Solution}

- Hypothesis from previous work that if $f_{n} \square \frac{d(\text { speed) }}{d t}$, then closed-form (computationally fast) standard analytical equation for SDOF steady-state response would be accurate.

$$
x_{\text {steady-state }}=\frac{A / \omega^{2}}{\sqrt{\left(1-\left(\frac{\Omega}{\omega}\right)^{2}\right)^{2}-\left(2 \zeta \frac{\Omega}{\omega}\right)^{2}}}
$$

- Validation by comparing response with numerical solution.

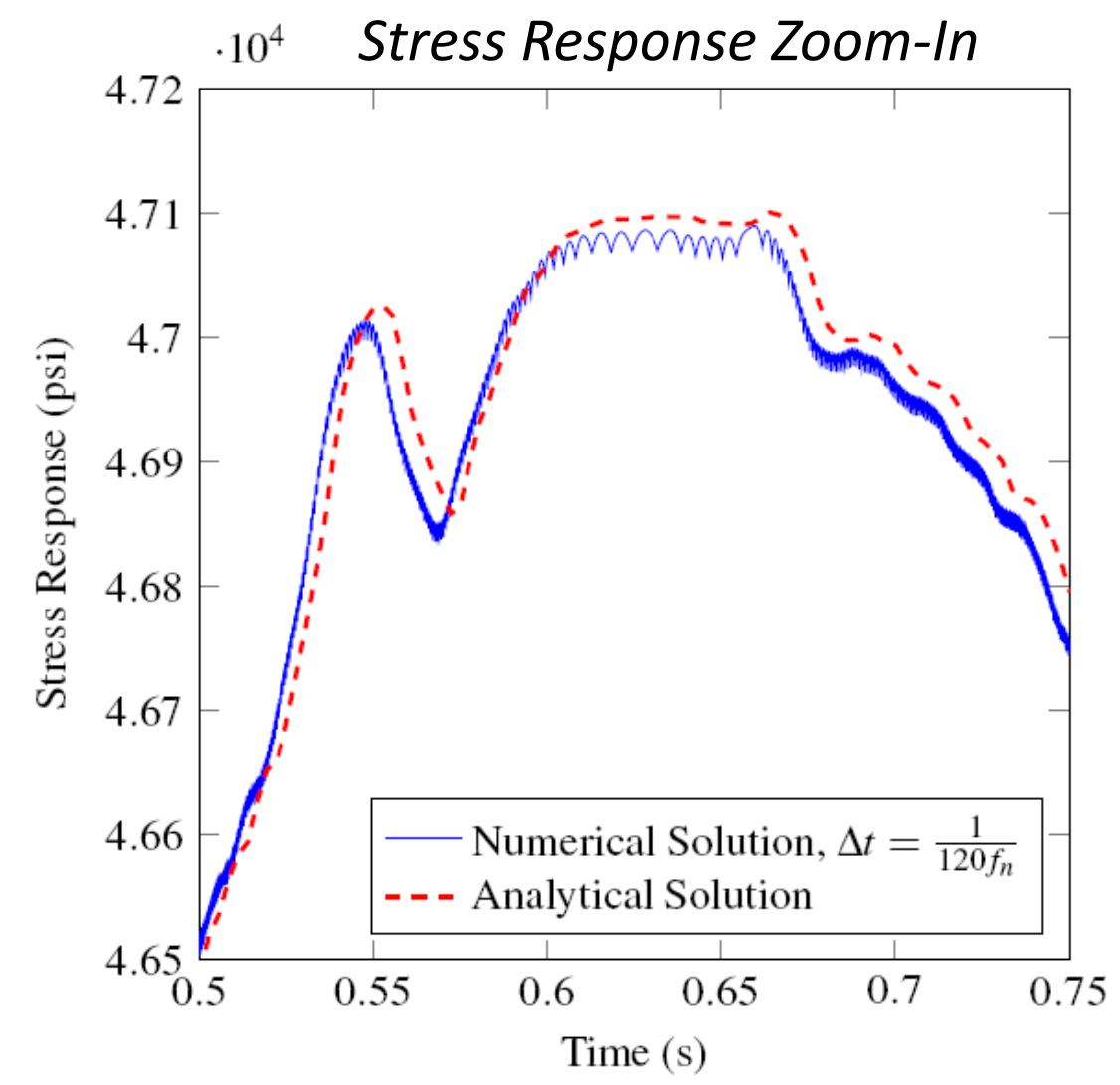




\section{Analytical Solution Validation}

- Validation also shown in damage accumulation plot; error in analytical steady-state method is $<1 \%$ ( $\Delta t \leq 1 / 120 f_{n}$ required).

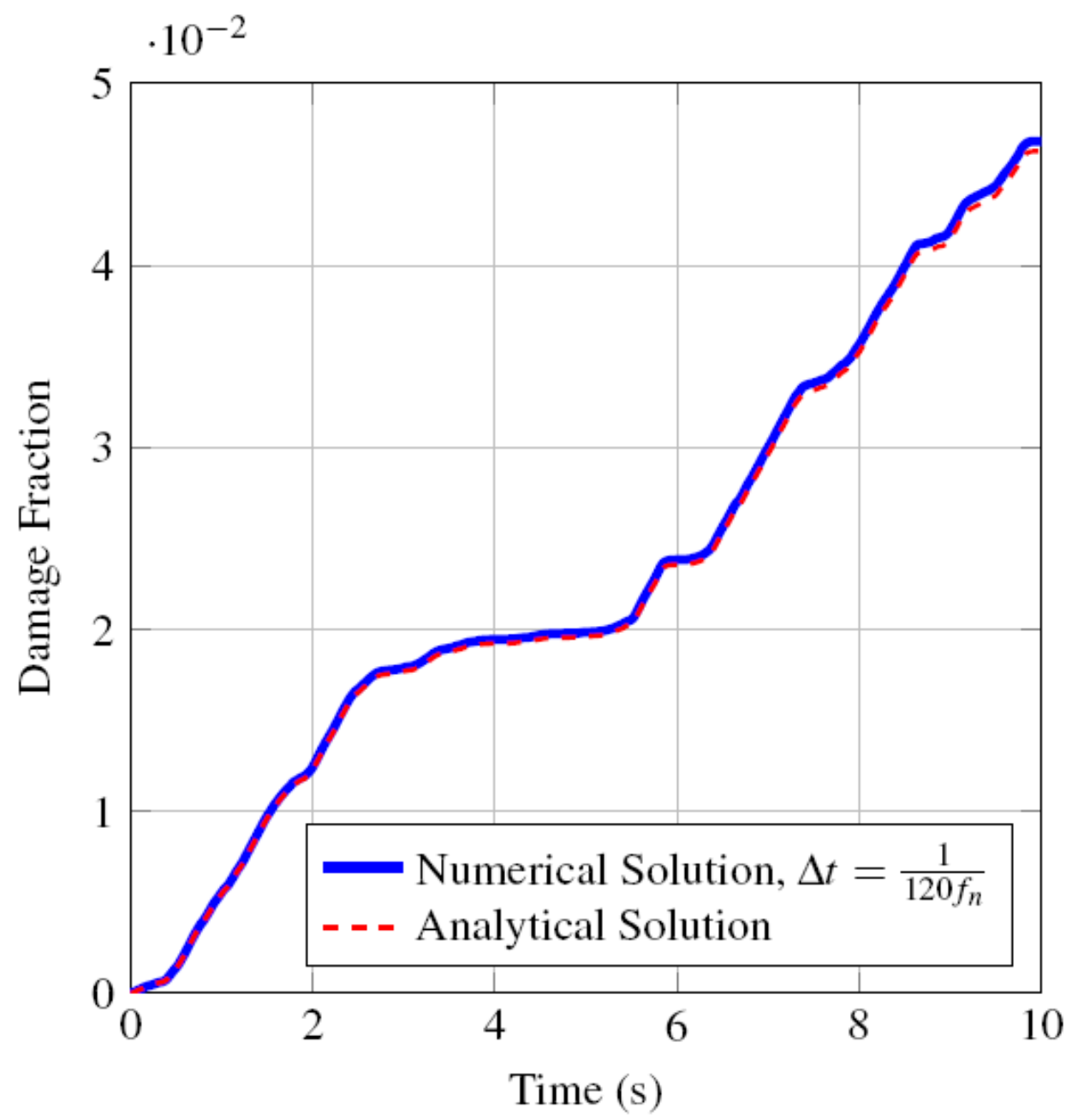


- This assumption good with high FPR, driving $f_{n} / \frac{d(\text { speed })}{d t}$ ratio up.

- FFT of speed shows mostly below $100 \mathrm{hz}$, very low compared with natural frequency.

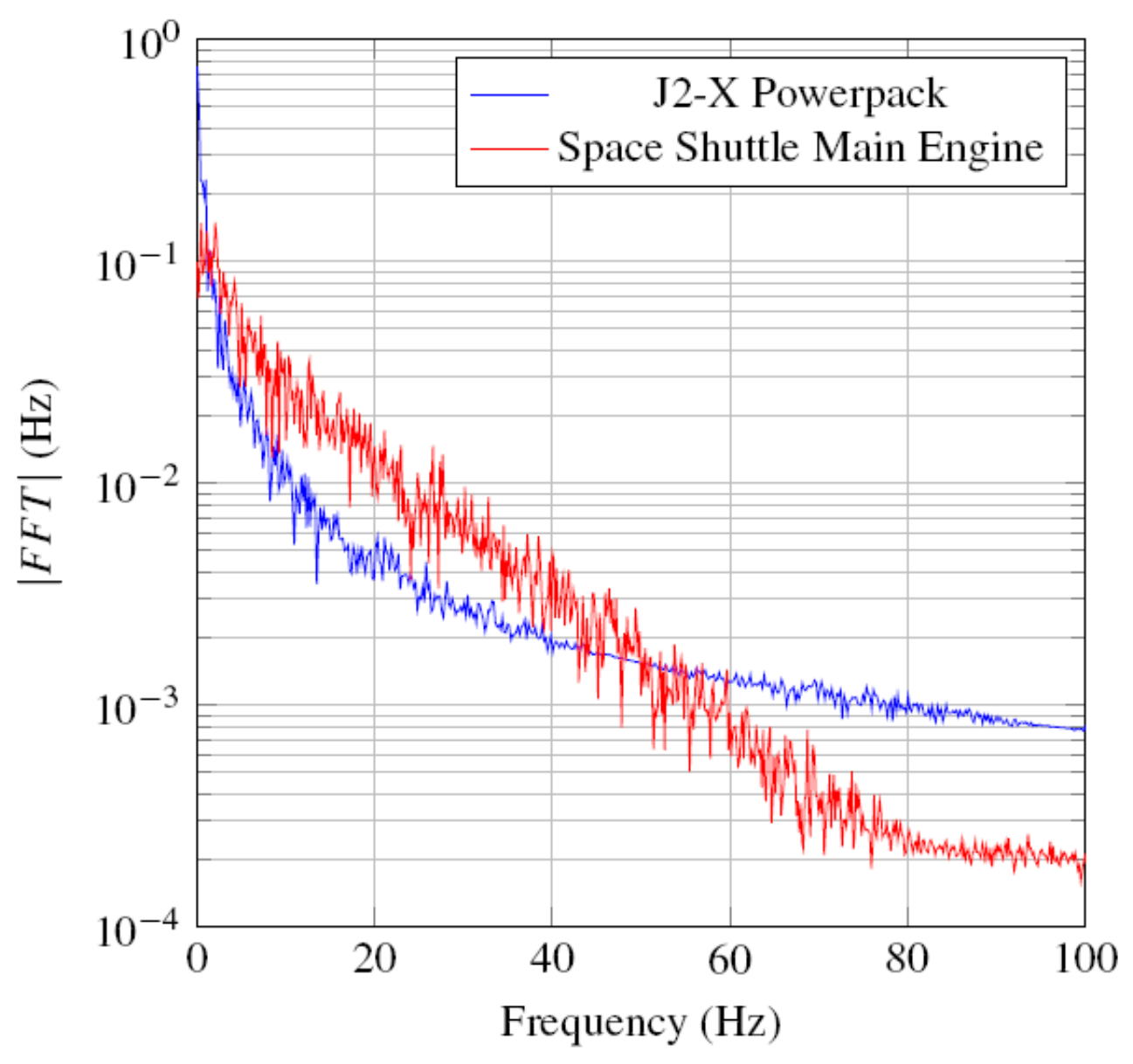


- Calculation of damage performed considering dither for specific $10 \mathrm{sec}$. window.

- Damage calculation assuming constant resonant excitation $\rightarrow$ 2.135 times more damage, call it "Dither Life Ratio".

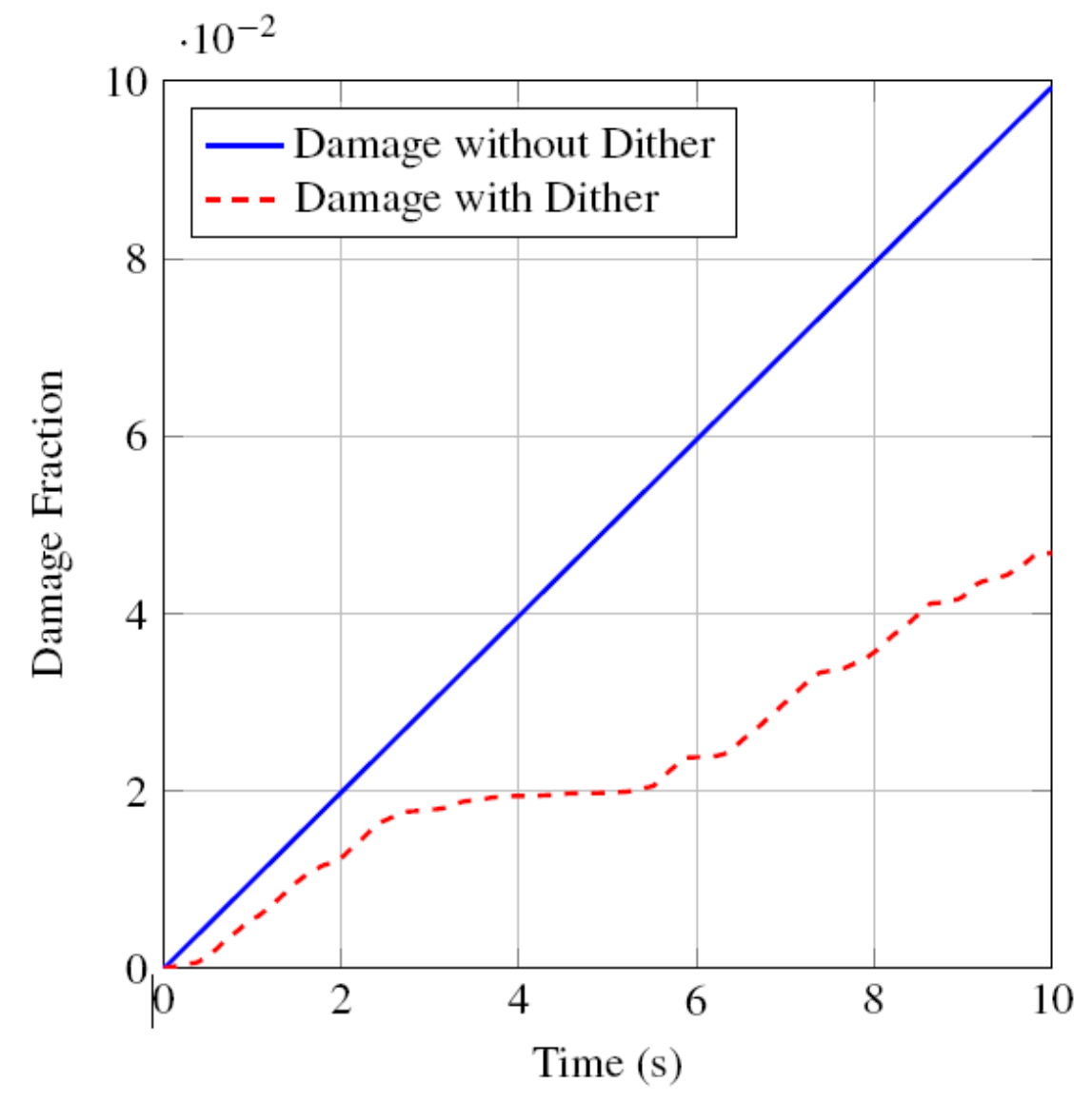


- During design phase, actual speed time histories unknown, but statistics from similar engines known.

- Prompted development of Monte Carlo method using rapid analytical solution.

- Speed vector created using Normal statistical distribution.

- Powerpack data $\rightarrow$ std dev $=38.6 \mathrm{hz}$ (cov=0.129\%).

- MC results linear because rate of change of frequency variation not correct (and very high), but damage accumulation is accurate on the average.

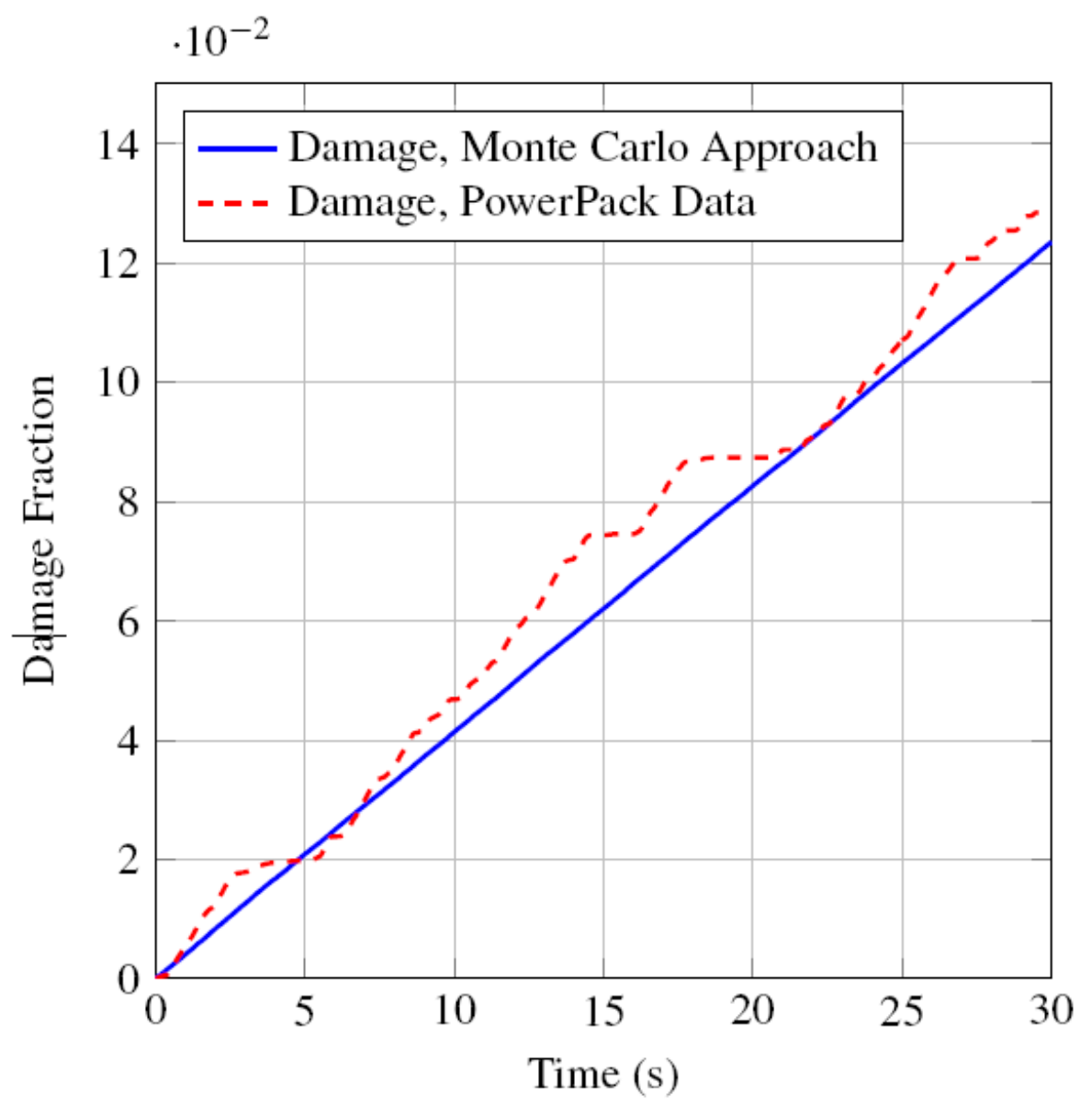




\section{Sensitivity of DLR to speed COV and $\zeta$}

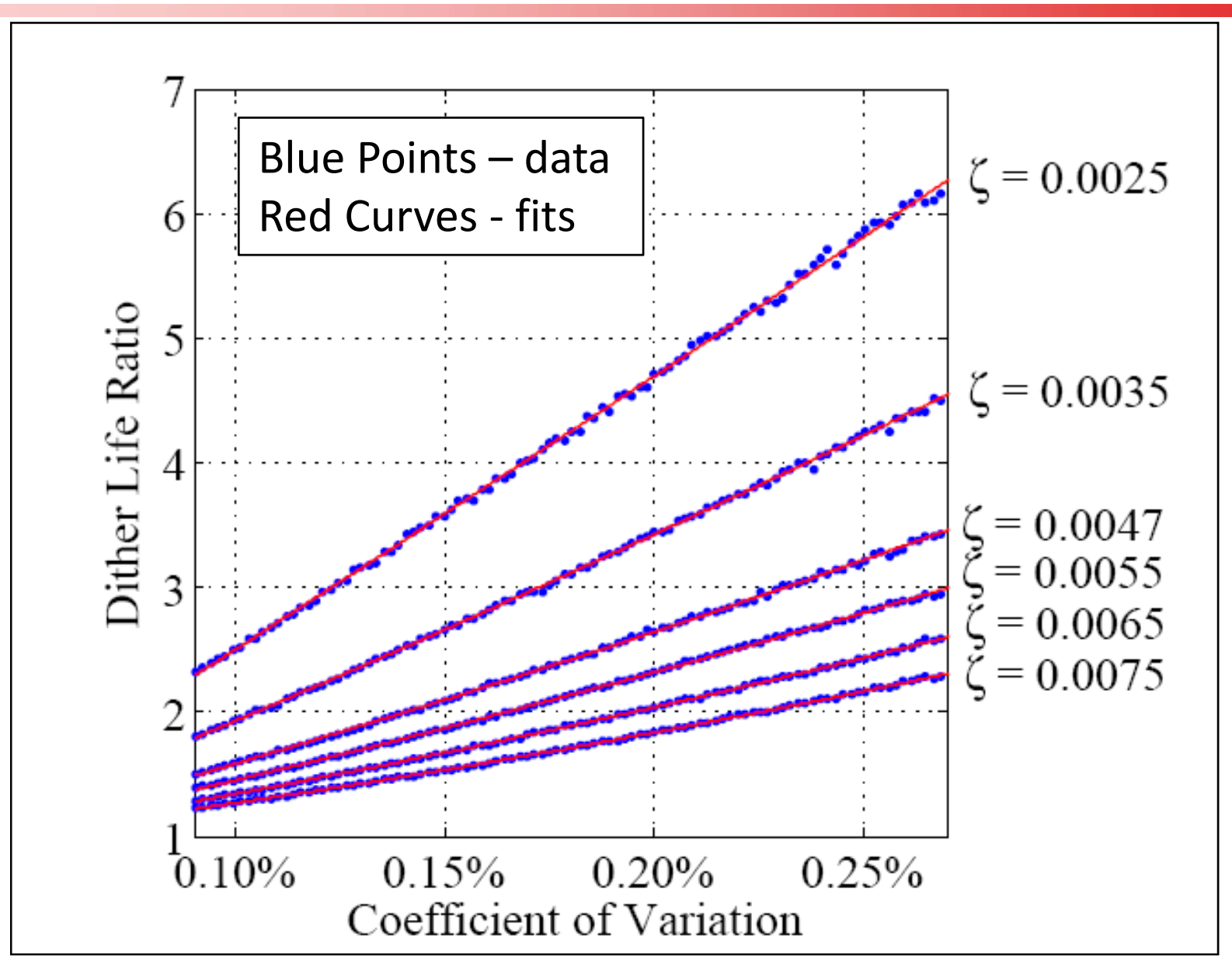

- Larger for high COV for speed, since more time spent off-resonance.

- Larger for small $\zeta$, since peaks are sharper and time spent offresonance will have less response. 


\section{Conclusions}

- Numerical and Analytical methods developed to determine damage accumulation in specific engine components when speed variation included.

- Dither Life Ratio shown to be well over factor of 2 for specific case.

- Steady-State assumption shown to be accurate for most turbopump cases, allowing rapid calculation of DLR.

- If hot-fire speed data unknown, Monte Carlo method developed that uses speed statistics for similar engines.

- Application of techniques allow analyst to reduce both uncertainty and excess conservatism.

- High values of DLR could allow previously unacceptable part to pass HCF criteria without redesign.

- Given benefit and ease of implementation, recommend that any finite life turbomachine component analysis adopt these techniques. 